\title{
Resolving Atomic Scale Chemistry and Structure at NO and Ba Passivated $\mathrm{SiC} / \mathrm{SiO}_{2}$ Interfaces
}

J. Houston Dycus ${ }^{1}$, Weizong $\mathrm{Xu}^{1}$, Daniel J. Lichtenwalner ${ }^{2}$, Brett Hull ${ }^{2}$, John W. Palmour ${ }^{2}$, and James M. LeBeau ${ }^{1}$

1. Department of Materials Science and Engineering, North Carolina State University, Raleigh, North Carolina 27695, USA

2. Power Devices R\&D, Wolfspeed, a Cree Company, 3028 E. Cornwallis Road, RTP, North Carolina 27709, USA

Silicon carbide has become a commonly used wide-bandgap material for power devices. A major challenge for SiC MOSFET device processing has been to reduce the density of trap states at the $\mathrm{SiC} / \mathrm{SiO}_{2}$ interface, which greatly reduces the inversion-layer field effect mobility, $\mu_{\mathrm{FE}}$, Nitric oxide (NO) annealing is commonly performed to passivate the $\mathrm{SiC} / \mathrm{SiO}_{2}$ interface and reduce the interface trap density. A new method for increasing $\mu_{\mathrm{FE}}$ involves depositing a layer of $\mathrm{Ba}$ at the interface which has been shown to produce $\mu_{\mathrm{FE}}$ values up to $85 \frac{\mathrm{cm} 2}{V^{*}}$ [1], more than double that with the NO anneal. The impact of the $\mathrm{SiC} / \mathrm{SiO}_{2}$ interface on $\mu_{\mathrm{FE}}$ has driven research towards studying the transition layer between $\mathrm{SiC}$ and $\mathrm{SiO}_{2}$ [2]. Although studies report chemical segregation of $\mathrm{N}$ in $\mathrm{NO}$ annealed devices [3], the influence of $\mathrm{Ba}$ is unreported. Further, the influence of interfacial treatments on the atomic structure remains unclear even for NO annealed devices.

In this talk, we will examine the impact of interfacial treatments on the $\mathrm{SiC} / \mathrm{SiO}_{2}$ interface using scanning transmission electron microscopy (STEM), electron energy-loss spectroscopy (EELS) and energy dispersive X-ray spectroscopy (EDS). From high angle annular dark-field (HAADF) imaging, we clearly resolve the $\mathrm{SiC} / \mathrm{SiO}_{2}$ interface for $\mathrm{Ba}$ and $\mathrm{NO}$ treated samples, Figure 1 (a-b). We will discuss $\mathrm{Ba}$ and NO interfaces, emphasizing the differences in the transition layer between each sample. Although HAADF STEM proves useful for Z contrast imaging, many ambiguities at interfaces remain without support from spectroscopy[4]. By employing EDS we will resolve such ambiguities and reveal the chemical makeup of each interface, Figure $1(\mathrm{c}, \mathrm{d})$. We will show that EDS reveals information at the interface that would be hidden by relying on HAADF imaging alone especially in the case of NO annealed devices.

We will then discuss the effects of interfacial chemistry on the atomic structure at the interface. Using Revolving STEM (RevSTEM) to correct drift and image distortion in STEM images, we obtain images with atom columns at highly accurate positions [5-7]. By measuring the atom column-to-atom column distances, we generate strain maps for each sample [8]. We will compare these results with geometric phase analysis (GPA) to address the strain states using multiple methods. For NO, there is a tensile strain within $\mathrm{SiC}$ at the $\mathrm{SiO}_{2} / \mathrm{SiC}$ interface, Figure 2. The $\mathrm{Ba}$ interlayer sample, however, exhibits no observable strain relative to the bulk $\mathrm{SiC}$ structure. We will discuss the bonding configuration and consequences of this lattice strain on electrical properties [9]. 


\section{References:}

[1] D. J. Lichtenwalner et al, Applied Physics Letters, 105(18) (2014).

[2] T. Zheleva et al, Applied Physics Letters, 93(2) (2008).

[3] K.-C. Chang et al, Journal of Applied Physics, 97(10) (2005).

[4] J. H. Dycus et al, Applied Physics Letters, 102(8) (2013).

[5] X. Sang and J. M. LeBeau, Ultramicroscopy, 138 (2014), p. 28-35.

[6] X. Sang et al, Microscopy and Microanalysis, 20 (2014), p. 1764-1771.

[7] J. H. Dycus et al, Microscopy and Microanalysis, 21 (2015), p. 946-952.

[8] A. A. Oni et al, Applied Physics Letters, 106(1) 2015.

[9] JHD, WX and JML acknowledge the Analytical Instrumentation Facility (AIF) at North Carolina State University. JHD acknowledges support by the National Science Foundation Graduate Research Fellowship (Grant DGE-1252376). Research was sponsored by the Army Research Laboratory under Cooperative Agreement \#W911NF-12-2-0064.

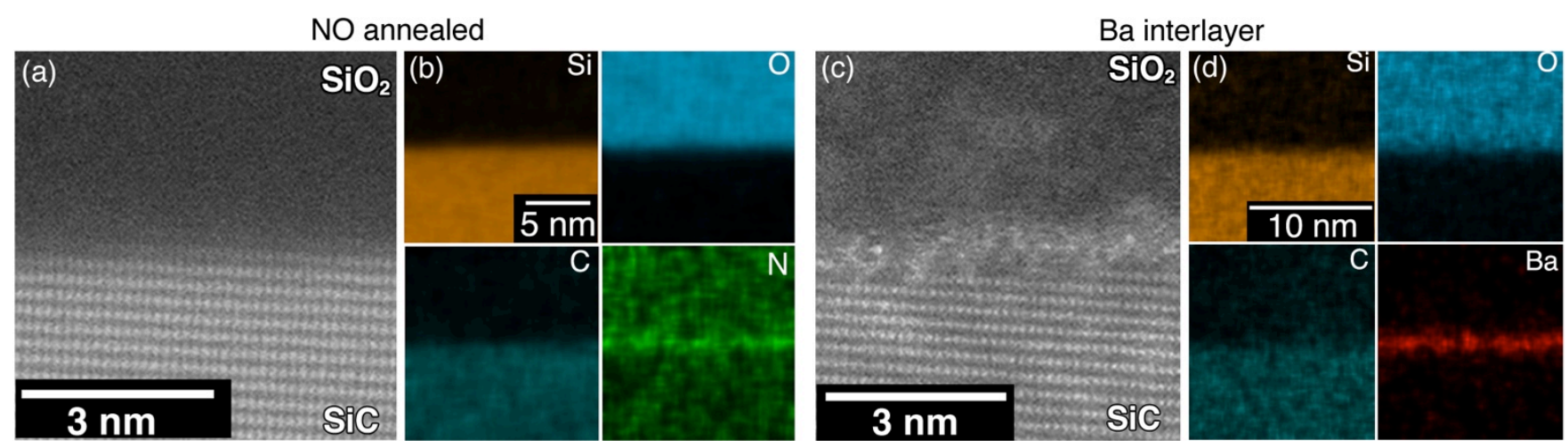

Figure 1: HAADF STEM of the $\mathrm{SiC} / \mathrm{SiO}_{2}$ interface when $\mathrm{NO}$ (a) and $\mathrm{Ba}$ (c) passivated. EDS maps from $\mathrm{NO}(\mathrm{b})$ and $\mathrm{Ba}(\mathrm{d})$ devices with elemental X-ray maps for all elements observed during acquisition.

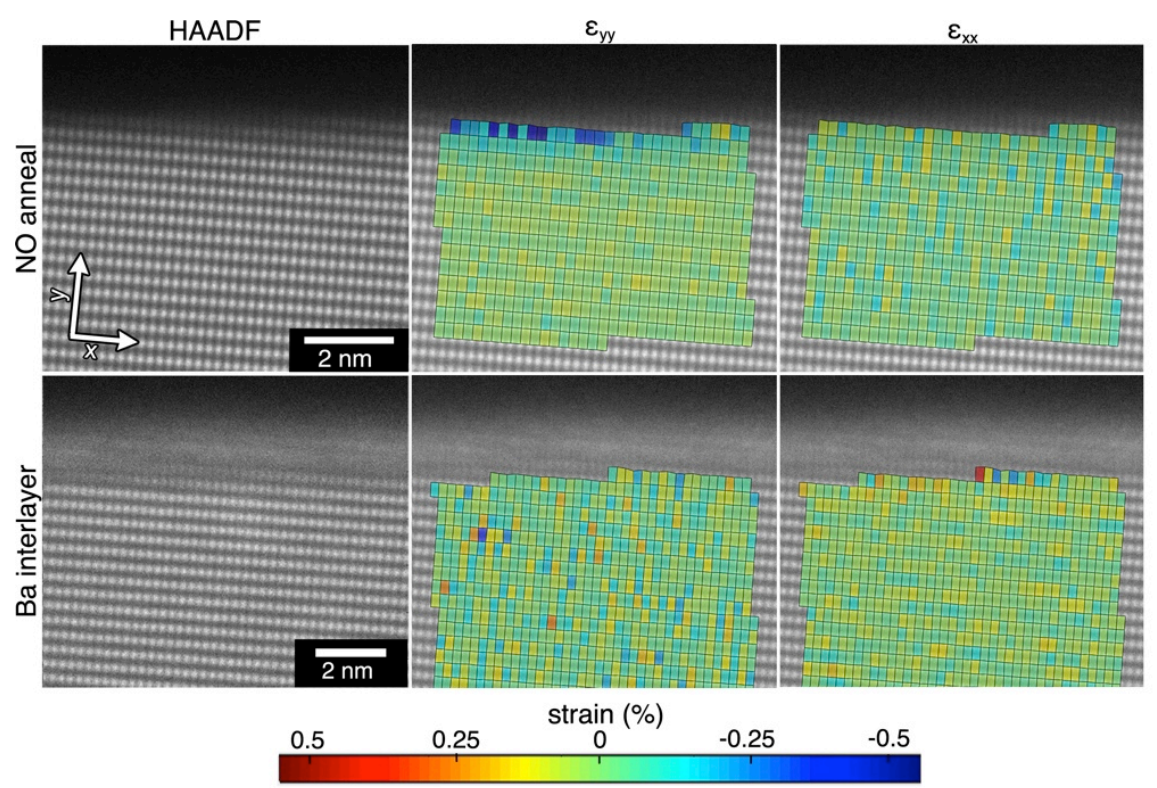

Figure 2: RevSTEM frame averaged series for both Ba (bottom left) and NO (top left) samples. Strain maps are overlaid on the RevSTEM images with values corresponding to the colorbar. 